\title{
FACTORES DE RIESGO CARDIOVASCULAR EN TRABAJADORES DE UNA FUNDACION, CARTAGENA
}

\section{CARDIOVASCULAR RISK FACTORS IN A FOUNDATION WORKERS, CARTAGENA}

TÍTULO CORTO: FACTORES DE RIESGO CARDIOVASCULAR EN TRABAJADORES

\author{
Amparo Astrid Montalvo-Prieto ${ }^{1}$, Johana Barcinilla-Alarcón ${ }^{2}$, Elindis Navarro-Guzmán ${ }^{3}$
}

Recibido en marzo 22 de 2016

Aceptado en agosto 08 de 2016

\section{RESUMEN}

La enfermedad cardiovascular es uno de los mayores problemas de salud pública en el mundo, por ser incapacitante y costosa. Se considera un trastorno de origen multifactorial, agrupados éstos en modificables y no modificable. Identificar los factores de riesgo cardiovascular en los trabajadores de una fundación, Cartagena. Se recolectó la información a 50 trabajadores de la Fundación objeto de estudio, mediante el procedimiento STEPS recomendado por la OMS, que permite diligenciar información sociodemográfica, mediciones físicas y bioquímicas. Los resultados se presentan utilizando la estadística descriptiva. Hubo una tasa de respuesta del 99,8 \% del total de los participantes. El promedio de edad de los participantes fue de 30.48 años, el $84 \%$ consume alcohol y verduras 4,54 días a la semana, bajo nivel de actividad física en el $76 \%$; sobrepeso en el $50 \%$ y el $12 \%$ presenta obesidad grado 2 , antecedentes de diabetes $44 \%$ y de hipertensión el $66 \%$. Determinar los factores de riesgo en etapas tempranas permitirá establecer acciones encaminadas a modificar estilos de vida y a disminuir el riesgo cardiovascular en los pacientes, además de promover el trabajo multisectorial para tomar decisiones y priorizar acciones.

Palabras clave: Factores de riesgo; Cerebrovascular; Cardiovascular

\section{ABSTRACT}

Cardiovascular disease is one of the major public health issues in the world, because of its disabling, and costly condition. It is considered a multifactorial disorder, grouped into modifiable and non-modifiable causes. To identify risk factors for cardiovascular disease in employees of a foundation in Cartagena. Information was collected on 50 employees of the Foundation under study, following the WHO recommended procedure, which requires sociodemographic information, and physical and biochemical measurements. The results are presented using descriptive statistics. 50 surveys were conducted, with a response rate of $99.8 \%$. Study participants have an average age of 30.48 years old, regularly consume alcohol (84\%) and vegetable 4.54 days per week, low level of physical

1. Enfermera. Docente Universidad de Cartagena. Cartagena, Colombia. Correo: amontalvop1@unicartagena.edu.co

2. Enfermera. Clínica Crecer. Cartagena, Colombia. Correo: jobaralarcon_123@hotmail.com

3. Enfermera. Clínica Crecer. Cartagena, Colombia. Correo: sidnile11@hotmail.com 
activity (76\%); $50 \%$ overweight and $12 \%$ grade 2 overweight, history of diabetes $44 \%$ and $66 \%$ hypertension. The identification of risk factors in the early stages will establish actions aimed at changing lifestyles and decrease the number of patients identified with cardiovascular risk as well as promote multisectoral work to make decisions and prioritize actions.

Keywords: Risk Factors; Cerebrovascular Disorders; Cardiovascular Diseases

\section{INTRODUCCIÓN}

Según datos de la Organización Mundial de la Salud $\mathcal{S}$ (OMS) en el 2008, el $63 \%$ de los 57 millones de muertes que tuvieron lugar en el mundo por enfermedades no transmisibles (ENT) fueron por enfermedades cardiovasculares (ECV): Diabetes, cáncer y enfermedades respiratorias crónicas ${ }^{1}$. El 29\% de las muertes causadas por las ENT se presenta en personas menores de 60 años en países de ingresos bajos y medios, frente al 13\% de muertes por este tipo de enfermedades en países de ingresos altos.

Estilos de vida sedentarios provocan el crecimiento de las ENT y, en países en desarrollo, el mayor consumo de alimentos hipercalóricos y de productos como el tabaco, alcohol y comida basura, como consecuencia de campañas y de su disponibilidad ${ }^{1}$. Acciones de intervención en estos factores de riesgo comportamentales, limitaría el número de muertes por esta causa ${ }^{1}$. Las enfermedades cardiovasculares (ECV) son un trastorno de tipo vascular multifactorial y uno de los mayores problemas de salud pública en todo el mundo.

El factor de riesgo de las ECV se aplica a aquellos signos biológicos y hábitos adquiridos que se han encontrado con mayor frecuencia entre los enfermos de cardiopatía, permitiendo a los grupos poblacionales, la identificación del riesgo de presentar la enfermedad en un futuro inmediato $^{2}$. Se han clasificados los factores de riesgo para las ECV en: Modificables cambios en el estilo de vida y el uso de terapia farmacológica y no modificables la edad, el género y la herencia ${ }^{3}$. Anualmente las cardiopatías, los accidentes cerebrovasculares (ACV) y la diabetes generan pérdidas de miles de millones de dólares en el mundo ${ }^{1}$.

En Colombia, para el año 2012, la tasa de mortalidad por ECV fue de 29,34 por 100.000 habitantes. Las tasas de muerte por 100.000 habitantes se presentaron por encima de 35 en los departamentos del Valle del Cauca, Santander y Boyacá y 10 en el Vichada, en la Guajira y el Vaupés ${ }^{4}$. En Cartagena en el año 2012 el 29,7\% ( $n=885)$ de las muertes fueron por causas cardiovasculares; corresponde así una tasa del 91,5 por 100.000 habitantes, dato por encima al 69,7 registrado en el 2011. Las causas de defunción fueron el Infarto agudo de miocardio; el Choque cardiogénico, la Insuficiencia Cardiaca Congestiva; choque hipovolémico y la ECV Isquémica o Hemorrágica 5 .

El Riesgo Cardiovascular (RCV) se define como la probabilidad de desarrollar una enfermedad coronaria, accidente cerebrovascular o arteriopatía periférica (ECV) en un tiempo de 10 años y el factor de RCV corresponde a una característica biológica o comportamental presente en una persona sana y se relaciona con la presencia posterior de una Enfermedad Cardiovascular (ECV) ${ }^{6}$. La estimación del RCV global, evalúa y determina la importancia del factor para el desarrollo de una enfermedad cardiovascular o cerebrovascular, la estimación de este riesgo permite priorizar y determinar la necesidad de tratamiento del individuo y las metas a las cuales se debe llegar?

Existen diferentes tablas para medir el riesgo cardiovascular de acuerdo al tipo de población en estudio. Su utilización permite determinar la probabilidad de tener eventos como enfermedad cardiovascular, infarto de miocardio o muerte por enfermedad coronaria en un período de tiempo. Calcular el riesgo en las personas y el impacto que podría tener la modificación de éstos factores, determinaran las actividades de prevención cardiovascular (primaria o secundaria) a realizar7. Las acciones en el primer nivel de prevención evitarían la aparición de nuevos casos y en los pacientes que presentan un episodio cardiovascular se les realiza acciones del segundo nivel de prevención, para evitar el desarrollo de nuevos episodios de la enfermedad o su muerte. La hipertensión arterial, la dislipidemia (hipercolesterolemia o hipertrigliceridemia), el tabaquismo, la diabetes mellitus, el ser mayor de 55 
años (hombres) y 65 años (mujeres), son factores que se consideran de riesgo relevantes para el desarrollo de la $\mathrm{ECV}^{8}$.

Se consideran factores de RCV relevantes y modificables, la hipertensión arterial, la hipercolesterolemia y el tabaquismo; como modificables o controlables la diabetes, el sedentarismo, la obesidad o sobrepeso y el consumo excesivo de alcohol; pero la edad, el género y los antecedentes familiares de enfermedad coronaria prematura se consideran como factores de riesgo no modificables $^{8}$.

La OMS recomendó el método STEPS como el instrumento para la vigilancia de los factores de riesgo de morbilidad y mortalidad para las enfermedades crónicas. La utilización de esta herramienta permite fortalecer la vigilancia a este tipo de enfermedades ${ }^{9,10}$. Para el año 2010 se publica en Colombia el primer estudio ${ }^{11}$ utilizando la metodología estandarizada por la OMS para la vigilancia de los factores de riesgo de enfermedades crónicas y, en otro estudio $^{12}$, se asocia la presencia de factores de riesgo con las enfermedades crónicas no transmisibles.

No hay información acerca de la realización de este tipo de estudio en poblaciones pequeñas. En Cartagena, en una Fundación cuyo propósito es reducir la mortalidad infantil, mejorar la calidad de vida y la formación en actividades productivas de las madres adolescentes, laboran un total de 65 personas entre empleados, vinculados por contratos de prestación de servicios y voluntarios, los cuales se encuentran en edades entre 21 a 55 años. Se ha observado por el trabajo que desempeñan, el contenido de las tareas y la celeridad con que se delegan funciones que exigen resultados inmediatos, el corto tiempo para hacer pausas activas y alimentarse, lo que ha generado casos de hipo e hipertensión aislada, dislipidemias, sobrepeso, vértigos de naturaleza idiopática, trastornos del comportamiento, situaciones de estrés, disputas entre compañeros etc., que pueden ser causas determinantes para desarrollar enfermedades cardiovasculares.

Por tal motivo, en el 2012 se implementó un programa orientado a los trabajadores sobre promoción y prevención, el cual tuvo como objetivo realizar tamizajes de laboratorio, consulta nutricional y medición de índice de masa corporal (IMC), lo que permitió determinar la tendencia a padecer trastornos relacionados con sobrepeso, dislipidemias, malos hábitos de alimentación y falta de ejercicio en la mayoría de los funcionarios. No existen registros estadísticos del estado de salud de los trabajadores. Con el presente estudio se busca identificar los factores de riesgo cardiovascular en los trabajadores de una fundación en Cartagena.

\section{MATERIALES Y MÉTODOS}

Estudio descriptivo de corte transversal realizado a trabajadores de una fundación de Cartagena, que desearon participar y cumplieron con los criterios de inclusión. Se utilizó el método STEPS recomendado por la OMS ${ }^{13}$, el cual se desarrolla en tres etapas secuenciales que proporcionan pautas y material de apoyo para llevar a cabo la vigilancia de los factores de riesgo de enfermedades crónicas. El tiempo de recolección de la información fue de seis meses.

El instrumento STEPS sirvió para realizar la evaluación de los factores de riesgo, así: Recopilación de información sociodemográfica y conductual (comportamientos cotidianos y antecedentes familiares), toma de mediciones físicas con pruebas sencillas (estatura y peso para calcular el Índice de Masa Corporal (IMC), mediante consulta médica y de enfermería se determina la proporción de adultos que padecen sobrepeso, obesidad, o tienen la tensión arterial alta) y medición bioquímica mediante muestra de sangre (glicemia y perfil lipídico) ${ }^{9,10}$. Para el almacenamiento y análisis estadístico de los datos se utilizó el programa Excel y SPSS Versión 20.0 y la estadística descriptiva.

\section{Declaración sobre aspectos éticos}

Se consideró el estudio de riesgo mínimo para los participantes, fue aprobado por el comité de Investigaciones y autorizado por la institución de salud, se conservó el rigor ético y metodológico. Se contempló el consentimiento informado, la participación voluntaria y el manejo confidencial de la información ${ }^{14}$.

\section{RESULTADOS}

\section{Características sociodemográficas y Comportamiento diarios}

Del total de los trabajadores (65) que pertenecen a la planta de personal de la fundación objeto de estudio, el $75 \%$ de ellos firmaron el consentimiento informado y participaron en el estudio, el promedio de edad fue de 30.48 años, de ellos el $82 \%$ pertenecen al sexo femenino con un promedio de edad 30.27 y para los hombres fue de31.44 años. El 28\% estudió hasta la secundaria; estudios técnicos el $12 \%$ y estudios universitarios completos el $48 \%$. Con relación al estado civil el 54\% tiene una unión formal y el $38 \%$ son solteros (Tabla 1). 
Tabla 1. Características sociodemográficas de trabajadores de una Fundación, Cartagena, 2014

\begin{tabular}{|l|c|c|}
\hline \multicolumn{1}{|c|}{ Sexo } & N & $\%$ \\
\hline Femenino & 41 & 82,0 \\
\hline Masculino & 9 & 18,0 \\
\hline Escolaridad & N & $\%$ \\
\hline Primaria & 1 & 2,0 \\
\hline Bachiller & 13 & 26,0 \\
\hline Técnico & 6 & 12,0 \\
\hline Universitario incomp & 1 & 2,0 \\
\hline Universitario & 24 & 48,0 \\
\hline Posgrado & 5 & 10,0 \\
\hline Estado civil & $\mathrm{N}$ & $\%$ \\
\hline Casado & 20 & 40,0 \\
\hline Divorciado & 3 & 6,0 \\
\hline Separado & 1 & 2,0 \\
\hline Soltero & 19 & 38,0 \\
\hline Union libre & 7 & 14,0 \\
\hline Total & $\mathbf{5 0}$ & $\mathbf{1 0 0 , 0}$ \\
\hline
\end{tabular}

Ninguno de los participantes fuma actualmente, el 84 $\%$ de ellos consume alcohol, $72 \%$, lo hizo en el último año y $48 \%$ en el último mes principalmente los fines de semana, entre 1 a tres ocasiones (Tabla 2).

Tabla 2. Consumo de alcohol en trabajadores de una Fundación, Cartagena, 2014

\begin{tabular}{|l|c|c|}
\hline \multicolumn{1}{|c|}{ ¿Consume usted alcohol? } & $\mathrm{N}$ & $\%$ \\
\hline No & 8 & 16,0 \\
\hline Si & 42 & 84,0 \\
\hline Consumo durante el último año & $\mathrm{N}$ & $\%$ \\
\hline No & 14 & 28,0 \\
\hline Si & 36 & 72,0 \\
\hline Consumo durante el último mes & $\mathrm{N}$ & $\%$ \\
\hline No & 26 & 52,0 \\
\hline Si & 24 & 48,0 \\
\hline Ocasiones durante el último mes & $\mathrm{N}$ & $\%$ \\
\hline O-1 vez & 45 & 90.0 \\
\hline 2-3 veces & 5 & 10.0 \\
\hline Días de la semana/consumo & $\mathrm{N}$ & $\%$ \\
\hline Lunes a jueves & 2 & 4,0 \\
\hline Viernes & 2 & 4,0 \\
\hline Sábado y domingo & 20 & 40,0 \\
\hline No & 26 & 52,0 \\
\hline Total & $\mathbf{5 0}$ & $\mathbf{1 0 0 , 0}$ \\
\hline
\end{tabular}

Los trabajadores consumen en promedio 4,08 frutas por día y un promedio de 1.54 porción/día. El promedio día/ semana de consumo de verduras es de 4,54. Al consultar acerca del tipo de aceite utilizado en la preparación de las comidas, un $90 \%$ respondió que utilizan aceite vegetal, un $6 \%$ utiliza además mantequilla y solo un $4 \%$ utiliza aceite de soya (Tabla 3 ).

Tabla 3. Régimen Alimentario en trabajadores de una Fundación, Cartagena, 2014

\begin{tabular}{|l|c|c|}
\hline Días que consume frutas & $\mathbf{N}$ & $\%$ \\
\hline 0 a 2 & 16 & 32 \\
\hline 3 a 5 & 17 & 34 \\
\hline 6 a 7 & 17 & 34 \\
\hline Aceite utilizado al cocinar & $\mathrm{N}$ & $\%$ \\
\hline Margarina & 1 & 2 \\
\hline Soya & 1 & 2 \\
\hline Vegetal & 45 & 90 \\
\hline Vegetal + mantequilla & 3 & 6 \\
\hline Total & 50 & 100 \\
\hline
\end{tabular}

De los participantes, el $74 \%$ consideran que su trabajo no requiere de actividad física, pero un $30 \%$ manifiesta que maneja cargas dentro de sus labores diarias y el $76 \%$ del personal camina durante el día. Al analizar esta información el médico y la enfermera concluyeron que de acuerdo a las informaciones los participantes caminan en promedio 30 minutos por día. A la semana el $24 \%$ practica en promedio una hora de deporte en su tiempo libre y en el trabajo las labores son realizadas por los trabajadores de forma sentada principalmente (Tabla 4).

Tabla 4. Actividad física en trabajadores de una Fundación. Cartagena, 2014

\begin{tabular}{|l|c|c|}
\hline ¿Su trabajo requiere actividad física? & $\mathrm{N}$ & $\%$ \\
\hline No & 37 & 74,0 \\
\hline $\mathrm{Si}$ & 13 & 26,0 \\
\hline ¿Realiza deporte en su tiempo libre? & $\mathrm{N}$ & $\%$ \\
\hline No & 38 & 76,0 \\
\hline $\mathrm{Si}$ & 12 & 24,0 \\
\hline ¿Maneja cargas en el trabajo? & $\mathrm{N}$ & $\%$ \\
\hline No & 35 & 70 \\
\hline $\mathrm{Si}$ & 15 & 30 \\
\hline iCamina o maneja bicicleta? & $\mathrm{N}$ & $\%$ \\
\hline No & 12 & 24 \\
\hline $\mathrm{Si}$ & 38 & 76 \\
\hline
\end{tabular}


A los participantes les han tomado la presión arterial al menos una vez en su vida, a quienes le han tomado la presión arterial en el último año les reportó cifras tendientes a la hipotensión/hipertensión en el 12\% de ellos. El 8\% de los trabajadores informa que, ante los resultados, los profesionales de la salud le recomendaron disminuir el consumo de sal, y disminuir de peso, actividad física al $10 \%$ y al $6 \%$ tomar medicamentos para controlar la presión arterial (Tabla 5). Al 66\% de los participantes del estudio se les encontró cifras tensionales normales, al $32 \%$ en pre-hipertensión y un $2 \%$ con cifras tensionales entre 140-159 mm de Hg. el dato sistólico y entre 90-99 mm de Hg. los valores diastólicos.

Tabla 5. Detección de alteraciones de la presión arterial en trabajadores de una Fundación. Cartagena, 2014

\begin{tabular}{|l|c|c|}
\hline \multicolumn{1}{|c|}{ Hipertensión/Hipotensión } & $\mathrm{N}$ & $\%$ \\
\hline No & 41 & 82,0 \\
\hline $\mathrm{Si}$ & 9 & 18,0 \\
\hline Medición durante el último año & $\mathrm{N}$ & $\%$ \\
\hline No & 44 & 88,0 \\
\hline $\mathrm{Si}$ & 6 & 12,0 \\
\hline $\begin{array}{l}\text { Ha tomado medicamentos en últimos } \\
12 \text { meses }\end{array}$ & $\mathrm{N}$ & $\%$ \\
\hline No & 47 & 94,0 \\
\hline Si & 3 & 6,0 \\
\hline $\begin{array}{l}\text { Recomendación de reducción } \\
\text { consumo de sal }\end{array}$ & $\mathrm{N}$ & $\%$ \\
\hline No & 47 & 94,0 \\
\hline Si & 3 & 6,0 \\
\hline $\begin{array}{l}\text { Recomendación de disminución de } \\
\text { peso }\end{array}$ & $\mathrm{N}$ & $\%$ \\
\hline No & 46 & 92,0 \\
\hline Si & 4 & 8,0 \\
\hline Recomendación de realizar ejercicio & $\mathrm{N}$ & $\%$ \\
\hline No & 45 & 90,0 \\
\hline Si & 5 & 10,0 \\
\hline Total & 50 & 100,0 \\
\hline
\end{tabular}

Al menos una vez en su vida los participantes se han realizado pruebas de glucemia con resultados tendientes a la hipoglucemia/hiperglucemia en el $2 \%$. El profesional de la salud le recomendó al $16 \%$ de ellos, tomar medicamentos para su control, un régimen alimentario especial al $22 \%$, reducción en el peso al $18 \%$ y al $32 \%$ practicar ejercicios. Los resultados de las pruebas bioquímicas mostraron que el $96 \%$ se encuentra en el rango normal $(<100 \mathrm{mg} / \mathrm{dl})$ y al resto se les encontró valores promedio de 61,5 mg/dl (Tabla 6).

Tabla 6. Detección de alteraciones de la glucemia en trabajadores de una Fundación. Cartagena, 2014

\begin{tabular}{|l|c|c|}
\hline \multicolumn{1}{|c|}{ Hiper/Hipoglucemia } & N & $\%$ \\
\hline No & 49 & 98,0 \\
\hline Si & 1 & 2,0 \\
\hline $\begin{array}{l}\text { Tratamiento para manejo de } \\
\text { glucemia }\end{array}$ & $\mathrm{N}$ & $\%$ \\
\hline No & 42 & 84,0 \\
\hline Si & 8 & 16,0 \\
\hline Recomendación de dieta especial & $\mathrm{N}$ & $\%$ \\
\hline No & 39 & 78,0 \\
\hline Si & 11 & 22,0 \\
\hline Recomendación de bajar de peso & $\mathrm{N}$ & $\%$ \\
\hline No & 41 & 82,0 \\
\hline Si & 9 & 18,0 \\
\hline $\begin{array}{l}\text { Recomendación a realizar } \\
\text { ejercicio }\end{array}$ & $\mathrm{N}$ & $\%$ \\
\hline No & 34 & 68,0 \\
\hline Si & 16 & 32,0 \\
\hline Total & 50 & 100,0 \\
\hline
\end{tabular}

Fuente: Datos del estudio.

En la consulta realizada, los participantes informaron tener antecedentes de diabetes 44\% , 66\% hipertensión arterial, presencia de ataques cardiacos en el $26 \%$, cáncer en el $40 \%$ y alteraciones en el colesterol el $46 \%$ de ellos (Tabla 7).

Tabla 7. Antecedente familiares de los trabajadores de una Fundación. Cartagena, 2014

\begin{tabular}{|l|c|c|}
\hline \multicolumn{1}{|c|}{ Diabetes } & N & $\%$ \\
\hline No & 28 & 56,0 \\
\hline Si & 22 & 44,0 \\
\hline Hipertensión arterial & N & $\%$ \\
\hline No & 17 & 34,0 \\
\hline Si & 33 & 66,0 \\
\hline Ataques cardiacos & $\mathrm{N}$ & $\%$ \\
\hline No & 37 & 74,0 \\
\hline Si & 13 & 26,0 \\
\hline Cáncer & $\mathrm{N}$ & $\%$ \\
\hline
\end{tabular}


Continuación Tabla 7.

\begin{tabular}{|l|c|c|}
\hline \multicolumn{1}{|c|}{ Diabetes } & N & $\%$ \\
\hline No & 30 & 60,0 \\
\hline Si & 20 & 40,0 \\
\hline Hipercolesterolemia & N & $\%$ \\
\hline No & 27 & 54,0 \\
\hline Si & 23 & 46,0 \\
\hline Total & 50 & 100,0 \\
\hline
\end{tabular}

\section{Mediciones físicas}

Las medidas antropométricas del grupo en estudio muestran que el peso se encuentra entre $60 \mathrm{~kg}$ a $70 \mathrm{~kg}$ de peso, estatura entre $160 \mathrm{~cm}$ y $170 \mathrm{~cm}$. El promedio en las mujeres fue $65.2 \mathrm{~kg}$ y $161.1 \mathrm{~cm}$ de estatura y en los hombres de $72,5 \mathrm{~kg}$ y $169.6 \mathrm{~cm}$; la frecuencia cardiaca promedio fue de 81 latidos por minuto y el del perímetro abdominal de $80 \mathrm{~cm}$.

Al establecer el índice de masa corporal se encontró en el grupo de estudio un $10 \%$ de personas obesas grado $1 \mathrm{y}$ $2 \%$ en obesidad grado 2 ; pero este dato aumenta en un $50 \%$ al observar el porcentaje de personas con sobrepeso.

\section{Resultados Pruebas Bioquímicas}

Los resultados de las pruebas bioquímicas realizadas, los valores promedio para el colesterol total fue de 202.9, el HDL promedio fue de $53.2 \mathrm{mg} / \mathrm{dl}$ (valores normales $>40$ y $<60 \mathrm{mg} / \mathrm{dl}$ ). El $38 \%$ de los participantes obtuvo valores promedio de colesterol LDL de $128.1 \mathrm{mg} / \mathrm{dl}$ y el $28 \%$ obtuvo valores mayores de $60 \mathrm{mg} / \mathrm{dl}$ y triglicéridos en $127.7 \mathrm{mg} / \mathrm{dl}$. Al evaluar los niveles de glucemia el $97 \%$ de los participantes mostró valores promedio de 76.6.

\section{DISCUSIÓN}

Identificar los factores de riesgo para eventos cardiovasculares y calcular su distribución en una población supone un reto, dado el marcado impacto que esto representa en la prevención de estas patologías, las cuales se consideran como causantes de millones de decesos en el mundo. La población del presente estudio presentó un promedio de edad de 30,48 años, condición que se comparte con diferentes estudios ${ }^{15,16}$ quienes reportan edades promedio de 33.25 y 33.5 años respectivamente y con relación al sexo, el primer estudio se realizó en hombres ${ }^{15} \mathrm{y}$ en el segundo estudio ${ }^{16} \mathrm{el}$
$72 \%$ fueron mujeres, dato este último que se acerca al reportado en la presente investigación. El consumo de alcohol como uno de los comportamientos consultados, se encontró que la información hallada en este estudio (48\%) es diferente a lo encontrado por Sirit et a ${ }^{15} \mathrm{y}$ Alayón et al ${ }^{16}$ quienes informan consumo de alcohol en sus participantes $91,6 \%$ y $11 \%$ respectivamente. Las diferencias de los resultados obtenidos en cuanto a consumo de alcohol pueden deberse a la presencia de un alto porcentaje de mujeres en el presente estudio, quienes además iniciaron el consumo de alcohol a edad temprana. Este dato se constituye en un alto factor de riesgo.

El consumo de cigarrillo como factor de riesgo cardiovascular no está presente en los participantes del presente estudio, similares resultados al hallado en otro estudio $^{15}$, pero contrario al estudio ${ }^{16}$ que reporta al $11 \%$ como fumadores. La condición de no fumadores para los participantes del presente estudio puede atribuirse a las fuertes campañas acerca del consumo de cigarrillo como factor de riesgo de enfermedades cerebro-cardiovasculares, el estar trabajando en la prestación de los servicios de salud y a las drásticas medidas presentes en la institución acerca de mantener ambientes libres de humo.

Los participantes del estudio consumen frutas y verduras diariamente, la literatura reporta el impacto en la prevención de RCV por este hábito alimentario ${ }^{17}$, contrario a lo descrito ${ }^{16}$, donde los participantes consumen dietas con alto consumo de grasas saturadas, ácidos grasos trans, colesterol y carbohidratos y escaso consumo de frutas y verduras. Existe evidencia de la asociación entre el consumo de frutas y verduras y la disminución del RCV, por tal razón es necesario realizar educación a la comunidad acerca de la importancia de la práctica de hábitos saludables e implementar en la dieta el consumo de alimentos con alto contenido de fibra y antioxidantes, ya que se ha asociado a una mejoría de algunos de los parámetros que modifican el RCV, como el colesterol total, colesterol LDL y la presión arterial.

Se destaca el sedentarismo en el $76 \%$ de los participantes, dato similar a lo obtenido en la Encuesta Nacional de Salud ${ }^{18}$ de $87.5 \%$, el estudio ${ }^{19,20}$ reportó en el 9,6\% de sus participantes la presencia de factores simultáneos de riesgo, como inactividad física y consumo agudo de alcohol y en el 6,9\% inactividad física, consumo agudo de alcohol y tabaco. La baja actividad física encontrada en este estudio fue superior a la reportada de 51,6\% y $57,4 \%{ }^{21,22}$. El 76\% de los participantes realizan algún tipo 
de actividad física durante el tiempo libre, dato similar a lo encontrado en otros estudios de $76,2 \%{ }^{18,21}$. Se encontró obesidad en el $10 \%$ de los participantes, dato inferior a lo reportado por Manzur y Arrieta ${ }^{2}$ de $11.8 \%$, pero si a este sobrepeso se suma la medida del perímetro abdominal que fue de $80 \mathrm{cms}$ promedio ${ }^{22}$, el riesgo es mayor, porque la acumulación de grasa a nivel visceral se considera factor predictor de enfermedades cardio-metabólicas.

Los antecedentes familiares como factor de riesgo no modificable son similares a los reportado en la literatura ${ }^{16}$, donde se muestra antecedentes como hipertensión arterial en el $73.3 \%$, hipercolesterolemia $46.6 \%$, diabetes mellitus $45 \%$, infarto agudo al miocardio $26.6 \%$ y cáncer en el $38.3 \%$. Contrario a estos datos ${ }^{23}$, los participantes del estudio presentaron obesidad en un $28.3 \%$, HTA $11,6 \%$, diabetes mellitus $2.2 \%$ y $1.4 \%$ infarto agudo de miocardio, lo que constituye la herencia como factor no modificable, en una condición de alta prevalencia en la población, pero el mantener estilos de vida saludables y la realización de ejercicios contribuye a mitigar el impacto de ésta.

El promedio de edad de la población en estudio -30.4 años- ha podido influir en los resultados obtenidos sobre hipertensión arterial, $18 \%$, ya que esta es una patología frecuente en personas mayores de 55 años, similares datos (prevalencia de $14 \%$ ) encontró ${ }^{24}$ en una población más numerosa, Segura ${ }^{25}$ informa contrario a esta información que el 32.4\% está clasificados en estado de pre-hipertensión. Ante estas situaciones, la identificación de los factores de riesgo en etapas tempranas permitirá establecer acciones encaminadas a modificar estilos de vida y a disminuir el número de hipertensos.

Los datos de hipercolesterolemia en las pruebas bioquímicas realizadas a los trabajadores fueron similares a lo encontrado por otros investigadores ${ }^{26} \mathrm{y}$ en cuanto a los valores de triglicéridos estuvo por debajo del valor reportado de $40.6 \%$; contrario a la prevalencia de $18,3 \%{ }^{27}$. Con relación a los niveles de glucemia el $97 \%$ de la población mostró valores dentro del rango normal; resultado similar encontrado por investigadores ${ }^{28}$. Éstos resultados pueden ser consecuencia de los estilos de vida presentes en la población en estudio.

\section{CONCLUSIÓN}

A pesar de ser una institución con una población de trabajadores pequeña, los resultados del estudio permiten llamar la atención acerca de la necesidad de realizar acciones de promoción y prevención a este grupo. El promedio de edad encontrado en los participantes del estudio fue de 30.4 años, población joven que facilita la intervención en cuanto a cambios de estilos de vida saludables.

La identificación de los factores de riesgo en etapas tempranas permitirá establecer acciones encaminadas a modificar estilos de vida y a disminuir el número de pacientes diagnosticados con hipertensión. El realizar intervenciones educativas acerca del consumo de frutas y verduras y de la de la práctica de hábitos saludables diariamente disminuirá en esta población los RCV.

Las condiciones laborales limitan aspectos como la actividad física debido a que por el tipo de actividad laboral y/o labores administrativas que propician el sedentarismo, sin embargo, un grupo apreciable realizan un promedio de una hora/semana de actividad física o deporte.

Los factores de riesgo para enfermedad cardiovascular encontrados en el grupo en estudio, son, consumo de alcohol, sedentarismo, en menor proporción sobrepeso u obesidad, prehipertensión y la herencia familiar. Modificar los estilos de vida y la realización de ejercicios contribuye a mitigar el impacto de la herencia sobre los mismos.

\section{AGRADECIMIENTOS}

A Eliana Bernal, William López, Álvaro Murgas por su participación en el presente estudio.

\section{DECLARACIÓN SOBRE CONFLICTO DE INTERESES}

Los autores no reportan conflictos a declarar relacionados con la investigación.

\section{REFERENCIAS BIBLIOGRÁFICAS}

1. Organización Mundial de la Salud. Informe sobre la situación mundial de las enfermedades no transmisibles. Resumen de orientación. Ginebra: OMS; 1994. (Consultado 2015 marzo 23). Disponible en: http:// www.who.int/nmh/publications/ncd_report_summary_ es.pdf

2. Manzur F, Arrieta C. Estudio sociológico y del conocimiento de los factores de riesgo de las enfermedades cardiovasculares en la Costa Caribe Colombiana (Estudio Caribe). Rev. Col. Cardiol; 2005;12(3): 122-8. 
3. Pearson TA, Blair SN, Daniels SR, Eckel RH, Fair JM, Fortmann SP, et al. AHA guidelines for primary prevention of cardiovascular disease and stroke. 2002 Update; 106:388-91

4. Centro de Innovación, Fundación Santa Fe de Bogotá. Seguimiento al Sector Salud en Colombia. Así vamos en Salud. [internet]. (Consultado 2016 julio 17). Disponible en: http://www.asivamosensalud.org/inidicadores/ estado-de-salud/grafica.ver/26

5. Alcaldía Mayor de Cartagena. DEPARTAMENTO ADMINISTRATIVO DISTRITAL DE SALUD - DADIS. Perfil epidemiológico de Cartagena 2012. Informe grupo epidemiológico. (Consultado 2015 marzo 23). Disponible en: http://www.minsalud.gov.co/sites/rid/ Lists/BibliotecaDigital/RIDE/VS/ED/PSP/ASIS \% 20 Cartagena \% 202012.pdf

6. Jackson R. Guidelines on preventing cardiovascular disease in clinical practice. BMJ 2000; 11;320(7236): 659-661.

7. Vélez S. Evaluación del riesgo cardiovascular global: una necesidad. Rev. Col. Cardiol. 2006 13(3): 139-40.

8. Chobanian AV, Bakris GL, Black HR, Cushman WC, Green LA, Izzo JL, et al. Seventh report of the Joint National Committee on Prevention, Detection, Evaluation, and Treatment of High Blood Pressure. Hypertension. 2003; 42(6):1206-52. (Consultado 2015 marzo 23). Disponible en:http://www.ncbi.nlm.nih.gov/pubmed/14656957

9. Organización Mundial de la Salud. Factores de riesgo de enfermedades cardiovasculares: nuevas esferas de investigación. Informe de un Grupo Científico de la OMS. Versión Panamericana del Instrumento STEPS. Ginebra: OMS; 1994. (Consultado 2015 marzo 23). Disponible en: http://www.who.int/chp/steps/instrument/PAHO_ STEPS_Instrument_v2.1_ES.pdf

10. Organización Mundial de la Salud. El Manual de vigilancia STEPS de la OMS: el método STEPwise de la OMS para la vigilancia de los factores de riesgo de las enfermedades crónicas. Ginebra, Organización Mundial de la Salud; 2006. (Consultado 2015 marzo 23). Disponible en: http://whqlibdoc.who.int/ publications/2006/9789244593838_spa.pdf

11. Secretaría de Salud de Santander, Observatorio de Salud Pública de Santander. Factores de riesgo para enfermedades crónicas en Santander, método STEPwise. Informe Grupo Científico de la Secretaría de Salud de Santander y del Observatorio de Salud Pública de Santander. Bucaramanga 2011. (Consultado 2014 marzo 1). Disponible en: http://www.who.int/chp/ steps/2010_STEPS_Survey_Colombia.pdf

12. Lopera V, Santacruz S. Factores de riesgo asociados a enfermedades crónicas no trasmisibles en Medellín en 2011. Evaluación con la metodología encuesta STEPwise. Revista Salud Pública de Medellín; 2012, 5(2): 63-78.
13. Organización Mundial de la Salud. Manual de vigilancia STEPS de la OMS. [internet]. (Consultado 2014 febrero 26). Disponible en: http://www.who.int/chp/steps/ Parte1.pdf

14. Colombia, Ministerio de salud. Resolución 8430 de 1993. Por el cual se establecen las normas científicas, técnicas y administrativas para la investigación en salud. Bogotá 1993.

15. Sirit Y, Acero C, Bellorin M, Portillo R. Síndrome Metabólico y otros Factores de Riesgo Cardiovascular en Trabajadores de una Planta de Policloruro de Vinilo. Rev. Salud pública. 2008; 10(2): 239-49.

16. Alayón AN, Ariza S, Baena K, Lambis L, Martínez L, Benítez L. Búsqueda activa y evaluación de factores de riesgo cardiovascular en adultos jóvenes, Cartagena de Indias, 2007. Biomédica. 2010; 30(2):23844. (Consultado 2015 marzo 23). Disponible en: http://www.scielo.org.co/scielo.php?pid = S012041572010000200010\&script $=$ sci_arttext

17. Castillo A, Smith C, Hirsch S, Brito A. ¿Es efectiva la consejería para aumentar el consumo de frutas y verduras y disminuir el riesgo cardiovascular en prevención secundaria? Una revisión. Rev chil nutr. 2008; 35(2):123-9.

18. Colombia. Ministerio de la Protección Social. Encuesta Nacional de Salud 2007. Resultados Nacionales. Bogotá: Javegraf; 2009. (Consultado 2015 marzo 23). Disponible en: http://www.minsalud.gov.co/Documentos $\% 20$ y\%20Publicaciones/ENCUESTA \%20NACIONAL.pdf

19. Gómez LF, Samper B, Cabrera G, Espinosa G, Mateus JC, Gómez LC. Factores de riesgo cardiovascular en la localidad de Santa Fe de la ciudad de Bogotá. Resultados obtenidos en el área demostrativa CARMEN. Investig. segur. soc. salud. 2004; (6):11-29.

20. Colombia. Ministerio de la Protección social, Profamilia, Instituto Nacional de Salud, Bienestar Familiar, Coldeportes, Organización Panamericana de la Salud, et. Al. Encuesta Nacional de la situación nutricional en Colombia 2010 ENSIN. Informe Grupo Científico. Bogotá 2010.

21. Patiño F, Arango E, Quintero M, Arenas M. Factores de riesgo cardiovascular en una población urbana de Colombia. Rev. salud pública 2011; 3(3): 433-45.

22. National Cholesterol Education Program, National Heart, Lung, and Blood Institute, National Institutes of Health NIH Publication. Detection, Evaluation, and Treatment of High Blood Cholesterol in Adults (Adult Treatment Panel III). Third Report of the National Cholesterol Education Program (NCEP). 2002: 3143-21.

23. Giraldo J, Granada P, Zapata C, Rodríguez J, Cediel V, Martínez W, et al. Riesgo cardiovascular en docentes y administrativos de la Universidad Tecnológica de Pereira. 1ª. Ed., Pereira: Publiprint LTDA; 2010. 
24. Colombia, Ministerio de Salud. III Estudio nacional de Salud Bucal - ENSAB III, II. Estudio Nacional de factores de riesgo de enfermedades crónicas-ENFREC II. Tomo VI. Informe de un Grupo Científico. Ministerio de Salud, República de Colombia; 1999: 1-67. (Consultado 2015 marzo 23). Disponible en: http://docplayer.es/2147444Iii-estudio-nacional-de-salud-bucal-ensab-iii-ii-estudionacional-de-factores-de-riesgo-de-enfermedadescronicas-enfrec-ii.html

25. Segura L, Agusti R, Parodi J e Investigadores del estudio Tornasol. Factores de riesgo de las enfermedades cardiovasculares en el Perú. (Estudio TORNASOL) Revista Peruana de Cardiología. 2006; 32(2):82-128.

26. Díaz-Realpe J, Muñoz-Martínez J Sierra-Torres C. Factores de Riesgo para Enfermedad Cardiovascular en
Trabajadores de una Institución Prestadora de Servicios de Salud, Colombia. Rev. Salud Pública. 2007; 9(1):64-75.

27. Hernández J, Herazo Y, Valero M. Frecuencia de Factores de Riesgo asociados a Enfermedades Cardiovasculares en Población Universitaria Joven. Rev. Salud Pública. 2010; 15(5): 852-64.

28. Lizarazu I, Rossi C, Iglesias J, Villanueva D. Prevalencia de factores de riesgo cardiovascular y evaluación del riesgo cardiovascular global en trabajadores de la Universidad Libre seccional Barranquilla, 2010 (Colombia). Salud Uninorte. 2013; 29(1): 52-63. (Consultado 2015 marzo 23). Disponible en: http:// rcientificas.uninorte.edu.co/index.php/salud/article/ view/4870/3317.

Para citar este artículo: Montalvo-Prieto AA, Barcinilla-Alarcón J, Navarro-Guzmán E. Factores de riesgo cardiovascular en trabajadores de una Fundación, Cartagena. Duazary. 2017 enero; 14 (1): 61 - 69 